\title{
Se confronter à la mort et l'apprivoiser
}

Jean Martin

\begin{abstract}
«Doctors \& Death» est un programme de SWIMSA (association des étudiants en médecine de Suisse), s'intéressant à ce qui se passe autour de la mort dans le monde de la santé. «Nous commençons nos études, dit leur site Web, par l'anatomie sur des cadavres, ce qui ne laisse personne indifférent. Les étudiants sont ensuite confrontés à la vie en clinique, avec les pronostics sombres, les accidents, les colloques en équipe, les familles et... les patients. Difficile de devenir un professionnel de santé et de se confronter à la mort, la souffrance, la rupture.»
\end{abstract}

\section{«Confrontés au corps mort, nous avons voulu réfléchir}

\section{au sens à donner à notre vécu.»}

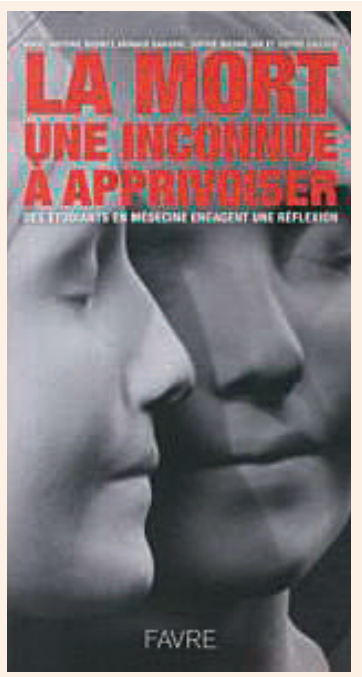

Marc-Antoine Bornet et al. (dir. publ.)

La mort: une inconnue à apprivoiser

Lausanne: Editions Favre; 2013

132 pages. $15.70 \mathrm{CHF}$

978-2-8289-1388-5

Des projets ont été développés dans ce cadre par des groupes d'étudiants de Berne et Lausanne. Les animateurs de l'initiative lausannoise disent dans l'introduction à leur publication: «Confrontés au corps mort [par les séances de dissection], nous avons voulu réfléchir au sens à donner à notre vécu. Rapidement, nous avons compris que ce n'était que la première marche d'un face à face avec la mort qui nous accompagnera tout au long de notre expérience médicale. Rester seul face à ces expériences n'est pas la solution aux interrogations devant ces territoires inconnus, ces peurs, ces doutes. C'est pourquoi nous avons sollicité différentes personnes pour qu'elles partagent avec nous leurs impressions lors de ces moments forts.»

Le résultat est un livre attrayant de 132 pages. Vingt-deux chapitres courts par des auteurs très divers: les quatre étudiants responsables du projet, des infirmières dont une personnalité médiatique romande (Rosette Poletti), une aumônière d'hôpital, une variété de huit médecins de disciplines et âges divers, des responsables d'enseignement d'anatomie et de sciences sociales. Avec une perspective historique de Vincent Barras, de l'Institut d'histoire de la médecine de Lausanne et en conclusion une lettre de P. - A. Michaud, Vice-doyen de la Faculté.

Grands thèmes abordés: 1) l'expérience de la dissection dans le cursus des étudiants en médecine et son rôle; 2) la mort, les mourants et les professionnels dans la pratique médicale et des soins, notamment les aspects relationnels; 3) plus largement la problématique de la mort dans ses dimensions philoso- phiques (et spirituelles dans quelques textes), culturelles et sociétales.

\section{Vécu de la dissection}

De manière compréhensible, les étudiants surtout mettent l'accent sur cette expérience récente (encore que les auteurs médecins aussi évoquent leurs souvenirs plus ou moins lointains).

«Quand nous avons dû commencer la dissection d'un corps entier, ma priorité n'a pas été de me précipiter dans l'action, mais de remercier la personne ici présente. Je n'arrivais pas à me dire que ce n'était qu'un cadavre. J'ai donc murmuré «merci monsieur» et j'ai incisé du menton au sternum.» Un de ses collègues: «Maintenant la gratitude a pris la place du dégoût. Je suis reconnaissant envers ces personnes qui ont décidé de donner leur corps.»

Pratiquement: «Il a fallu s'approcher de notre table de dissection. C'était un peu comme faire connaissance avec quelqu'un, en l'occurrence notre cadavre. On l'a observé en entier, comme pour l'apprivoiser.» Un autre: «En sentant la résistance de la peau sous la lame de mon scalpel, je me rendis brusquement compte que je découpais un corps humain et toutes mes idées auparavant si claires commencèrent à se bousculer dans ma tête.»

\section{Place de la dissection dans la formation - un rôle initiatique?}

Le Vice-doyen lausannois pour l'enseignement: «Force est de constater que l'enseignement de l'anatomie évolue: le développement fulgurant des techniques d'imagerie, l'apparition des technologies de simulation, tout cela oblige à réfléchir à la place qu'occupera à l'avenir l'enseignement de sciences de base dans le curriculum.» Concluant toutefois qu'«il y a dans l'enseignement des sciences fondamentales, y compris l'anatomie, une étape de formation de l'esprit indispensable».

Dans une autre contribution: «On pourrait à vrai dire, pour la plupart des futurs médecins, renoncer à la dissection, les moyens électroniques permettant d'apprendre le corps humain, y compris en 3D, sans disséquer.» Mais plusieurs relèvent un rôle de rituel initiatique des séances de dissection: «Ne faudrait-il pas voir dans cette singulière pratique un momentclé de la formation médicale, assimilé à un rite corporatiste, tissant chez les étudiants des liens particuliers», en rapport avec un enseignement qui a son côté caché, presque secret?... Un autre: «Entrée dans ces circonstances liées aux médecins qui en font des gens 
«différents〉, avec leurs prérogatives spécifiques. Filiation séculaire avec ceux qui ont voulu savoir comment le corps humain est 〈fabriqué»» (l'œuvre majeure de Vésale est intitulée De humani corporis fabrica).

\section{La mort - interactions entre malades et soignants/aidants}

Des interrogations fondamentales sur l'évolution nécessaire des attitudes au sein du corps médical et chez d'autres. Une responsable de gériatrie: «La mort restet-elle indéfiniment un échec de la médecine? Ou son acceptation et l'accompagnement du malade jusqu'à sa fin font-ils partie intégrante du projet thérapeutique que l'on construit avec lui?» Durant ses études et le début de son activité médicale, la mort n'avait jamais été présentée comme faisant partie d'un projet de soins. «On nous enseignait comment traiter les malades et repousser la mort [...]. Je souhaiterais qu'on m'ait parlé de la mort de mes futurs malades.» Souvent encore aujourd'hui, «on n'en parle pas, d'un commun accord tacite entre médecin et malade. Pourtant les malades y pensent souvent, quotidiennement peut-être.» Et plus loin: «Aborder la question de la mort nécessite un mariage subtil entre excellence clinique et intelligence de cœur.»

Tiré d'une recherche sociologique auprès de bénévoles: «Incontestablement, la confrontation aux patients mourants peut être l'occasion d'un échange, mais elle repose sur une base paradoxale: elle allie une forme d'empathie, d'une part, et d'altérité irréductible, d'autre part. L'un reste et l'autre part [...]. L'engagement des bénévoles se caractérise ainsi par une scission entre le souci réel qu'ils ont pour les patients et le souci qu'ils ont pour eux-mêmes, leur envie de vivre une expérience particulière.» N.B.: pas exclu que cette problématique touche aussi les professionnels.

Tiré d'un témoignage américain d'une élève infirmière mourante: «Je suis le symbole de votre peur de ce que nous savons que nous devons tous affronter un jour. J'ai conscience de votre peur et elle accroît la mienne. De quoi avez-vous peur? C'est moi qui meurs!»

Un étudiant, à propos d'un médecin suivant des patients sidéens: «ll nous a demandé combien d'entre nous s'étaient intéressés à l'anamnèse spirituelle de nos patients. Le silence gêné de l'assemblée en disait long sur la réponse.» Le même auteur, plus loin: «Comment pouvons-nous décider aujourd'hui qu'il est temps de laisser s'en aller nos patients ou nos proches? J'aimerais reprendre l'expression d'un médecin-chef qui disait en parlant des mourants «Il ne faut pas leur voler leur mort - dans un monde où la médecine apporte de plus en plus de solutions pour prolonger la vie mais oublie peut-être de reconnaître ses limites à cause de sa soif de maîtrise. Importance pour le médecin d'accepter un non-savoir et un laisser-être respectueux de l'être intime des sujets.»

Et des remarques quelque peu provocantes du

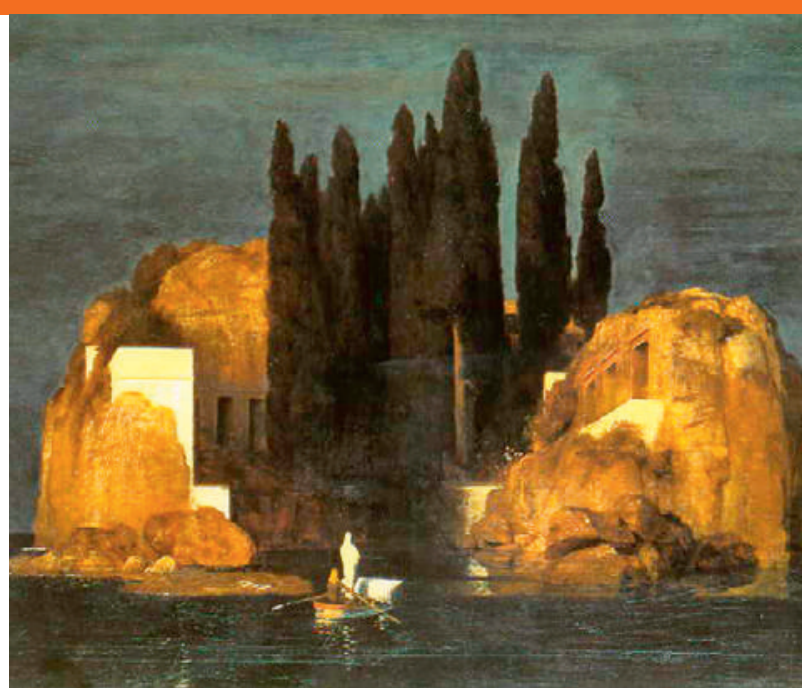

Un des jeunes auteurs du livre: «e souhaiterais qu'on m'ait parlé de la mort de mes futurs malades.» (L'lle des Morts d'Arnold Böcklin, 1880).

chef du service de soins palliatifs du CHUV: «On demande souvent aux médecins de soins palliatifs comment ils font pour supporter une telle activité professionnelle [...]. Or, c'est exactement l'inverse. Le travail en médecine palliative et dans l'accompagnement en fin de vie est un grand cadeau.» Plus loin: «En tant que médecins ou infirmiers en bonne santé, nous devrions nous garder de nous apitoyer sur les 〈pauvres malades». Car nous ne savons pas si ce n'est pas, en vérité, le contraire, à savoir que c'est nous qui sommes à plaindre et avons plus besoin de l'aide de nos patients qu'eux de la nôtre.»

\section{La mort dans la société}

Un médecin de service d'urgences: «De nos jours, s'il est incontestablement demandé au médecin de repousser les limites de l'existence humaine, ce à quoi il est largement formé, est-il pour autant préparé à assumer ce rôle de passeur, au sens mythologique du terme, que la société lui attribue de manière implicite?» Très bonne question. Avec une citation d'un texte évoquant le «rapport entre les mourants et les bien-portants, progressivement déplacé au point de ne plus concerner que le seul corps médical [...], la mort a été repoussée dans les coulisses de la scène sociale».

Dans l'article du directeur de l'Institut d'histoire de la médecine: «Le théâtre de la mort, qui s'est médicalisé au fil des deux derniers siècles au point qu'on a pu parler de confiscation de la mort par la médecine, est aujourd'hui marqué par la multiplication des acteurs. Ethiciens, politiciens, citoyens, etc. disputent plus que jamais à la médecine son droit exclusif d'intervention et d'expertise sur la mort.» Posant des questions interpellantes: «Dans quelle mesure par exemple faut-il comparer l'histoire des institutions de naissance (maternité, professions de l'obstétrique, procréation médicalement assistée) avec celle des institutions de mort (dissection, morgue, professions thanatologiques)». «Dans quelle mesure une thanatologie au sens strict du terme peut-elle prétendre au même statut épistémologique que la biologie, vu l'asymétrie fondamentale de leurs objets»? 